Michio Masujima

\title{
Applied Mathematical Methods in Theoretical Physics
}

Second, Enlarged and Improved Edition

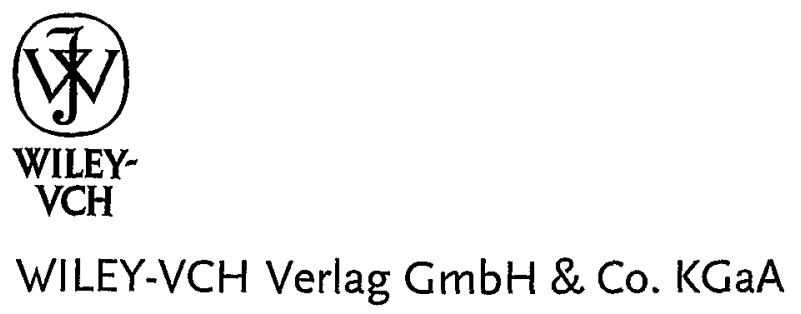




\section{Contents}

\section{Preface $X I$}

Introduction $X V$

1 Function Spaces, Linear Operators, and Green's Functions 1

1.1 Function Spaces 1

1.2 Orthonormal System of Functions 3

1.3 Linear Operators 5

1.4 Eigenvalues and Eigenfunctions 7

1.5 The Fredholm Alternative 9

1.6 Self-Adjoint Operators 12

1.7 Green's Functions for Differential Equations 14

1.8 Review of Complex Analysis 18

1.9 Review of Fourier Transform 25

2 Integral Equations and Green's Functions 31

2.1 Introduction to Integral Equations 31

2.2 Relationship of Integral Equations with Differential Equations and Green's Functions 37

2.3 Sturm-Liouville System 43

2.4 Green's Function for Time-Dependent Scattering Problem 47

2.5 Lippmann-Schwinger Equation 51

2.6 Scalar Field Interacting with Static Source 62

2.7 Problems for Chapter 267

3 Integral Equations of the Volterra Type 105

3.1 Iterative Solution to Volterra Integral Equation of the Second Kind 105

3.2 Solvable Cases of the Volterra Integral Equation 108

3.3 Problems for Chapter $3 \quad 112$ 


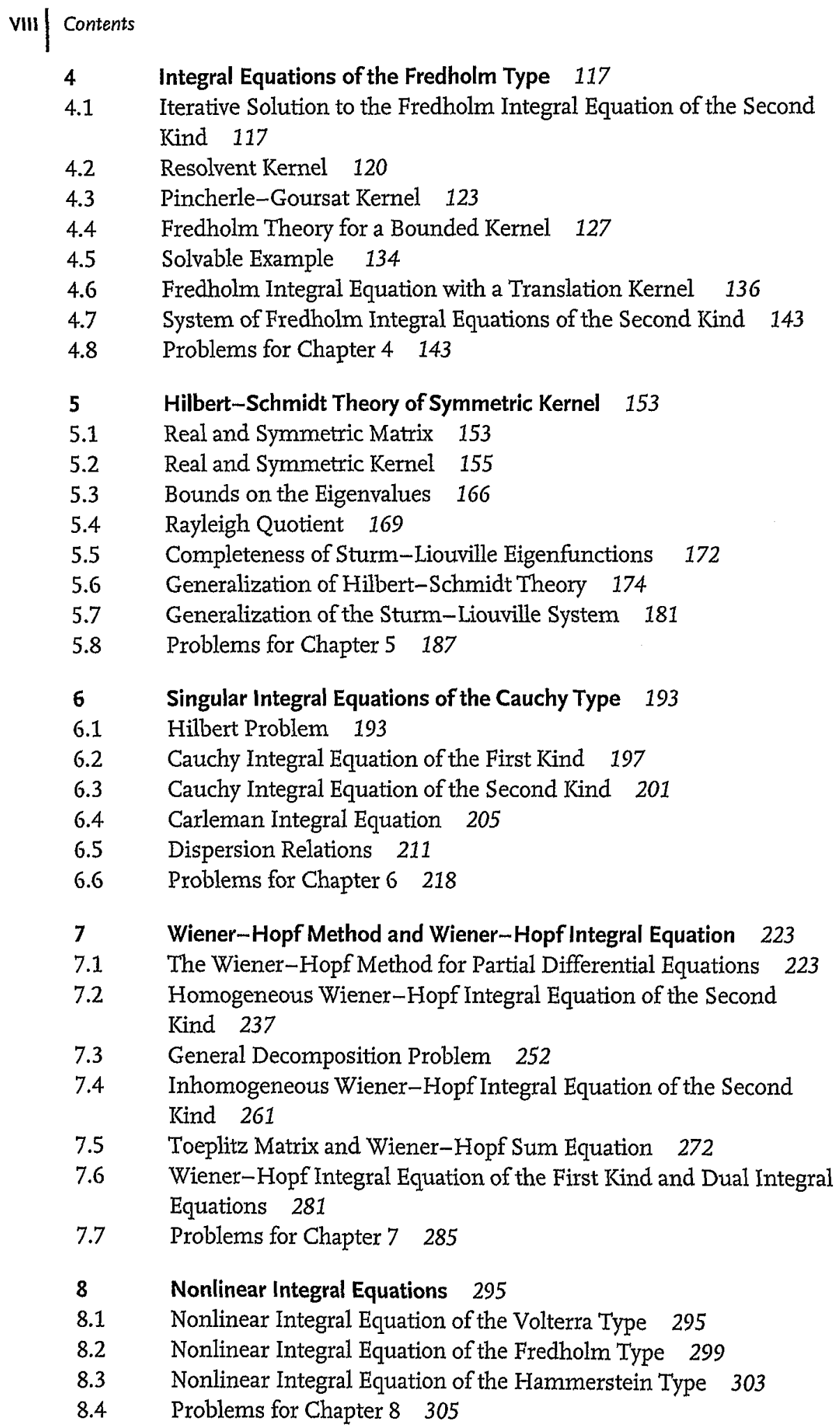


9 Calculus of Variations: Fundamentals 309

9.1 Historical Background 309

9.2 Examples 313

9.3 Euler Equation 314

9.4 Generalization of the Basic Problems 319

9.5 More Examples 323

9.6 Differential Equations, Integral Equations, and Extremization of Integrals 326

9.7 The Second Variation 330

9.8 Weierstrass-Erdmann Corner Relation 345

9.9 Problems for Chapter 9349

10 Calculus of Variations: Applications 353

10.1 Hamilton-Jacobi Equation and Quantum Mechanics 353

10.2 Feynman's Action Principle in Quantum Theory 361

10.3 Schwinger's Action Principle in Quantum Theory 368

10.4 Schwinger-Dyson Equation in Quantum Field Theory 371

10.5 Schwinger-Dyson Equation in Quantum Statistical Mechanics 385

10.6 Feynman's Variational Principle 395

10.7 Poincare Transformation and Spin 407

10.8 Conservation Laws and Noether's Theorem 411

10.9 Weyl's Gauge Principle 418

10.10 Path Integral Quantization of Gauge Field I 437

10.11 Path Integral Quantization of Gauge Field II 454

10.12 BRST Invariance and Renormalization 468

10.13 Asymptotic Disaster in QED 475

10.14 Asymptotic Freedom in QCD 479

10.15 Renormalization Group Equations 487

10.16 Standard Model 499

10.17 Lattice Gauge Field Theory and Quark Confinement 518

10.18 WKB Approximation in Path Integral Formalism 523

10.19 Hartree-Fock Equation 526

10.20 Problems for Chapter $10 \quad 529$

References 567

Index 573 